\title{
Improving handover of patient care using a new weekend proforma with a focus on ceiling of care
}

\author{
Christopher Yusuf Akhunbay-Fudge, Imogen Buss, Abigail Ward, Charlotte Snead, Miranda Cole, Archie Coulter
} North Bristol NHS Trust

\begin{abstract}
Patient handover is paramount for effective patient care and is often poorly documented or incomplete. North Bristol NHS Trust weekend handover proformas identify medical patients requiring weekend review. Many patients seen during on-call shifts are not handed over. Our aim was to develop Friday ward round proforma sheets for medical patients, to encourage clear documentation of management plans in order to improve handover of important information, particularly ceiling of care decisions.
\end{abstract}

Questionnaires were completed by F1 doctors regarding current handover systems. Baseline data collected by on-call F1s included time of understanding a patient's ceiling of care decision, and difficulty of comprehension of medical notes. Repeat data were collected with novel proformas in situ. Multiple cycles were performed to refine the sheets and target problems arising in their use.

Ninety-three percent of F1s wanted improved patient handover, with ceiling of care (87\%) and management plans (73\%) being the most difficult areas to understand. Time taken to ascertain ceiling of care decisions improved with the introduction of Friday handover proformas; mean time 153 seconds before and 5 seconds after. Clarity and documentation of management plans improved, with 50\% improvement in ease of understanding medical notes.

Results demonstrate that introducing Friday ward round proformas for medical patients improves communication between weekday and oncall teams, highlights current escalation of care plans, and leads to faster decision-making. Future plans include the introduction of a short educational session to the new F1 doctors and continued progress with introduction into hospital stationary.

\section{Problem}

The handover of patient information between doctors is often highlighted as an area of importance and a potential time for errors to occur.[1][2] Patient safety is vital in any hospital setting; encouraging concise yet comprehensive handover of care should aim to avoid such issues. This is particularly important with regards to weekend handover, which requires junior doctors to document clear plans for implementation over the following days and anticipate which patients will require regular medical review. The job of completing handover sheets often falls to the most junior members of the medical team, who lack training on the completion of these sheets.

The medical team handover in North Bristol NHS Trust (NBT), a tertiary referral centre in England, currently takes the form of a printed proforma for identifying patients that have outstanding investigations or require weekend reviews. The handwritten sheets are completed on Friday by doctors on each ward and filed in the office for the on-call team. This system for the handover for specific patients is in accordance to existing recommendations for use of written and standardised documentation for weekend handovers.[3][4]

However, this proforma is extremely brief, often failing to explain in adequate detail each patient's background and ongoing significant issues. There is some variety in the format of the handover sheets; they are often not uniform and may differ in the extent of completion. Furthermore, weekend ward cover usually involves reviewing a number of patients on the wards who become increasingly unwell and were not included in the weekend handover for review.[5]

This means that patient notes, particularly the most recent entries, are the primary source of patient information for the on-call team. Daily documentation by medical teams familiar with these patients can be inconsistent regarding the detail included, resulting in on-call doctors spending time filtering through patient notes to identify current problems and the management plan. Documented decisions such as the ceiling-of-care are frequently difficult to find, if present at all. This may cause unnecessary investigations or treatment, and further time spent in discussion with the intensive therapy unit (ITU).

Prior to the weekend, clearer documentation of patient's current issues, weekend plan, and ceiling of care would save time, improve continuity of care for patients, and reduce stress for junior doctors when dealing with unfamiliar patients.

\section{Background}

In recent years, the working patterns of doctors have increasingly moved towards shift work in response to hours restrictions both as part of the New Deal and European Working Time Directive (EWTD). As a result of this, patients are cared for by multiple 
different teams over the course of any given day or week, with handover between these different teams being of paramount importance.[2,5] The British Medical Association recommends the transfer of high quality information to involve time for teams to meet and discuss outstanding tasks, as well as up-to-date summaries and management plans for all patients.[2]

Handover of care has been highlighted as a patient safety issue, both as a result of poor communication and flaws in the system.[2,3,6] With regards to this, the General Medical Council has highlighted in "Good Medical Practice" the importance of continuity of care and handover of responsibility.[7] The importance of effective handover is being initiated into medical school teaching, and enhanced by the requirement of continuity of care as a Foundation Programme competency.[4,8]

There have been multiple published QI projects attempting to improve both handover and documentation. These demonstrated improvements in patient safety and doctor efficiency, although most interventions focussed on altering current weekend handover sheets in the relevant Trusts.[9-11] Only one published QI project concentrated on improving Friday ward rounds; it demonstrated improvement in documentation of escalation plans from $9 \%$ at baseline to $41 \%$ after introduction of new Friday ward round sheets.[12] Other sources highlight the usefulness of electronic components of handover, recommending its use in all Trusts to enhance task prioritisation and completeness of information for the handover.[9,13,14]

Within NBT, summary stickers for weekend handover are already in use among surgical firms. They are an effective manner in which to handover patient care, depending on the extent to which they are completed. We felt that a variation on these stickers would improve documentation on medical ward rounds prior to the weekend. Used in conjunction with the weekend handover sheets for specific patients and jobs, this should enhance handover of patient information.

\section{Baseline measurement}

Personal experience of working medical weekend shifts on-call demonstrated a wide variation in the quality of patient information being included on weekend handover sheets. Often during on-call shifts, doctors are asked to review patients who have not been formally handed over, leading to extra time spent ascertaining the current issues, management plan, and ceiling of care decisions for these patients. Weekend plan stickers for all surgical patients already exist, so the focus of the intervention remained on medical patients.

Our baseline aim was to establish the current issues with handover for weekend on-call shifts.

\section{First measurement}

A survey (Appendix 1, page 1) was devised and distributed to F1 doctors at NBT to gauge opinion on the weekend handover process and identify problems. The survey included four questions: two polar questions, one multiple choice, and one Likert-style question. Fifteen responses were obtained from a total number of $54 \mathrm{~F} 1$ doctors at the Trust, indicating a $27 \%$ response rate.

The escalation of treatment decisions was the most frequent area of concern identified by respondents (87\%). Closely following this was identifying the current management plan for patients, which $73 \%$ of respondents reported experiencing difficulty with. Ninety-three percent of respondents felt there was room for improvement in the format of handover, although only $53 \%$ felt their teams would be amenable to doing so, due to time restrictions. This information led the group to redirect the focus of the intervention specifically on the documentation of DNAR status and ceiling of care plans.

\section{Second measurement}

On-call F1 doctors over the weekend (both day and night teams) recorded all jobs performed on each ward and the time taken to understand the patient's medical history, current issues and management plan (Appendix 1, page 2). This was based on the time spent at the patient's notes, and the difficulty of understanding the notes, marked on a 10-point linear scale. This baseline measurement was conducted over two consecutive weekends.

The data was entered into a spreadsheet and analysed. The F1 doctors received 70 calls to see patients over both weekends. The mean time spent with patient notes was 570 seconds (range 60-1800) and the mean difficulty of understanding notes was 6.2 (range 2.1-9.3).

The length of time spent with patient notes varied a great deal depending on the job required, ranging from an average of 160 seconds to review an NG tube placement on chest $x$ ray, to 720 seconds for reviewing a patient with new symptoms. Our data showed that $47 \%$ of these patients requiring review at the weekend had not been included in the existing formal weekend handover. Following this we decided to narrow our intervention to primarily focus on one area of review of patient notes; the ceiling of care documentation.

\section{Third measurement}

The time taken to understand specifically ceiling of care decisions in patient notes was quantified in our third baseline measurement. Six patients' notes were examined on one ward, with the mean time taken to work out ceiling of care decisions found to be 153 seconds (range 53-300, median 103). The difficulty of determining a patient's ceiling of care was also quantified on the ten point linear scale, with a mean of 5.7 (Range 3.1-7.2).

See supplementary file: ds3737.docx - "Appendix 1 - Survey and baseline Questionnaire"

\section{Design}

We identified that on-call doctors spent a significant length of time on patient notes when trying to comprehend the current issues and management plans, and a significant proportion of these patients 
BMJ Quality Improvement Reports

were not formally handed over. We focussed on ceiling of care decisions; these are often poorly documented and a common issue causing concern when reviewing deteriorating patients on call.[12,15] It would be impractical to conduct a formal handover of every patient to the on-call team, therefore documentation in the patient notes must be up-to-date and easily accessible to the weekend staff.

Our intervention aimed to improve documentation of weekend plans and ceiling of care decisions. Therefore, a novel proforma sheet was designed, to prompt the documentation of key information (in place of normal continuation sheets) during the Friday ward round. It was designed to ensure that ceiling of care decisions are being reviewed and documented regularly. The team recognised the importance of the sheet being simple in layout, to ensure flexibility for use in different medical specialties. It also contains space for the minimum of three patient identifiers (as required by the Royal College of Physicians).[3]

We sought feedback from clinical directors throughout the development process, and they were highly supportive of the introduction of novel ward round sheets to assist in weekend handover of patient care. It was felt that either a bottom-up or topdown approach alone would not be sufficient to ensure the longevity of the intervention, leading us to involve both junior and senior doctors in the hope that the sheet would be used appropriately in order to benefit both patients and healthcare staff alike.

Nursing representatives were engaged throughout the design process of the proforma. Entries in the medical notes from ward rounds are a vital reference source for nursing staff regarding the current medical plan; a concise summary can be used to effectively deliver nursing care and respond to questions from concerned relatives. Feedback was provided by nurses, most of whom believed that the proformas would be a good idea, with the caveat that they are filled out correctly. Constructive criticism related to whether the proformas would be practical on wards with a high turnover of patients or complex patients.

The proformas were also presented to the patient records committee in order to gauge their opinions on our intervention as patients are ultimately the largest stakeholders in the intervention. The panel were largely supportive and were keen to take it forwards.

\section{Strategy}

PDSA cycle 1. A white proforma sheet was designed with prompts for all key information required to review unfamiliar patients, including:

\section{- Presenting complaint \\ - Important background}

\section{- Current issues}

- Ward round notes and plan

\section{- Ceiling of care}

- Tick box to complete if "not for resuscitation" decision already in place

- Tick box to complete if medically fit for discharge.

This information is not consistently documented as part of a preweekend patient summary, but is very important in allowing weekend staff to quickly familiarise themselves with an unwell patient's history.

The white proformas were filled out by doctors on a respiratory ward for a small group of patients after an explanation about their purpose. This ward was chosen because our second baseline measurement data revealed that junior doctors were frequently called to respiratory wards to assess unwell patients out of hours.

Two outcome measures were used to assess improvement with the proforma:

a) Length of time in seconds (from opening the notes) to identify ceiling of care for a patient

b) Difficulty of understanding the patient's case (recorded subjectively on a 10-point linear scale). Members of the weekend night team were asked to review patient notes both with and without the white proforma, and to document these outcome measures for each set.

PDSA cycle 2. The proforma was printed on green paper in order to be more easily identifiable. All other components of the sheet remained the same. Data were collected in the same manner and on the same ward as for PDSA cycle 1.

PDSA cycle 3. Weekend handover stickers are currently used for surgical patients in the Trust; these were discussed as a possible substitute for the proforma. However despite these being quick to fill out and easy to stick into the next section of the notes (rather than starting a new page), they would not provide space for documenting the necessary information and medical ward round notes. This may lead to duplication of information from the ward round notes on a Friday ward-round sticker, consuming valuable time. On the other hand, an A4 paper proforma could be filled in during the Friday ward round as a more structured and comprehensive documentation. Following discussion with junior doctors, it was decided that stickers would not provide benefit over paper sheets and would not be worth the additional printing expense.

PDSA cycle 4. During PDSA cycles 1 and 2 it became apparent that the new proformas were often filed in the incorrect section, rather than in chronological order with the current admission notes. This was probably because they were unfamiliar, different in design from the ordinary ward stationary, and had no continuation lines on the back. If this trend was to continue then it would defeat their purpose; important patient information would become difficult to find when required promptly. This unforeseen problem prompted a redesign of the proforma to make it appear more like the standard 


\section{BMJ Quality Improvement Reports}

hospital stationary, yet still stand out in the notes. To achieve this we changed the background colour of the sheet back to white, introduced continuation lines on the back, and placed an eyecatching bright yellow and black striped line down the right hand side. This newly designed proforma was introduced on the entire respiratory ward. As for cycles 1 and 2, the night F1 repeated the data collection from notes with and without the proforma. On the Monday we also recorded how many patients had weekend handover proformas completed (a possible indication of their ease of use). For patients who did not have proformas or had them incompletely filled out, we recorded reasons why this might have been the case.

PDSA cycle 5. The next cycle involved the addition of boxes for the date and doctor's signature on the proforma, as this was often forgotten.

PDSA cycle 6. The proformas were distributed again to an entire elderly care ward to determine flexibility and practicality of use. Prior to distribution, we educated the junior doctors on the ward about the purpose of our study and to how the proformas were to be filled in. Data were collected in the same manner as previous cycles.

See supplementary file: ds3736.pdf - "Final version of proforma"

\section{Post-measurement}

At baseline measurement it took approximately 153 seconds $(n=6)$ to understand ceiling of care decisions and "difficulty of understanding of patient notes" was relatively neutral (5.7). The introduction of plain white proforma sheets amongst a few patients reduced the time to find ceiling of care decision to 42 seconds $(n=$ 6 ) and difficulty of understanding notes changed to 3.5. This demonstrated more than a three fold improvement in the time taken to establish a patient's ceiling of care decision from their notes.

The introduction of green coloured proforma sheets instead of white slightly improved the time taken to understand ceiling of care decisions (mean $=33$ seconds $(n=5)$ ). The difficulty of understanding patient notes reduced to 0.8 .

Once the final proformas were designed, they were introduced on an entire ward. The time taken to establish the ceiling of care decision was further reduced (mean $=5$ seconds $(n=3)$ ). The difficulty of understanding was 2.9 (50\% improvement from baseline). Out of a total of 23 patients on the ward, three (13\%) had the proformas correctly completed and four $(17 \%)$ had them partially completed (ie no ceiling of care decision but DNAR decision completed). The reasons for partial completion were due to a medical student assisting with the ward round and writing in the notes, without being aware that the ceiling of care decision should be documented. Sixteen patients $(70 \%)$ did not have the proformas completed at all. The reasons for this were because the proformas were completed on a Friday afternoon, whilst the data collection was performed on Monday. By this time there were a substantial number of new patients on the ward who did not have proformas. Also, the medical registrar leading the ward round had been unaware of the plan to trial the proforma sheets.

This data collection cycle demonstrated a few issues currently preventing the use of the weekend handover proformas. Due to the fact that they are currently not in common use throughout the Trust, barriers exist due to poor knowledge of the use of new proformas and the fact that they are not in use on all wards. This problem should improve if the proformas are accepted into general hospital stationary, and then if patients are transferred between medical wards over the weekend, they should still have Friday ward round proformas in place.

In order to gain insight into whether the proformas are practical to be used on an entire ward every Friday, they were trialled again on a further medical ward, following an educational session. Out of a total of 15 patients, $100 \%$ of the proformas were filled in completely and establishing ceiling of care decisions took a mean of five seconds $(n=15)$.

See supplementary file: ds3712.docx - "Graphic representations of results"

\section{Lessons and limitations}

Weekend handover is a difficult problem to address given its subjective nature. Quality of handover was assessed by identifying a specific area of difficulty (ceiling of care) based on our initial survey, and then applying an objective measure (time taken to find this) in assessing the progress of improvement. Therefore, the results should be interpreted with caution when applied to other aspects of weekend handover.

Secondly, there was reluctance among junior doctors to accept an intervention which could potentially add to the workload of ward rounds; often the proformas were not being completed fully. In an attempt to resolve this, the proforma was designed to be easily integrated with the ward round by providing large areas for free text focusing on key information. Stickers were eliminated for this reason of creating additional work. We hope that the intended benefits of the proformas during on-call shifts would lead junior doctors to be more accepting of the slight alteration to the Friday work load.

Thirdly, the need for the proforma to be applicable to a wide variety of specialities within medicine was identified. The proforma's emphasis on free text and key information improves its diversity in clinical settings.

Finally, the weekend and night on-call shifts were often busy for F1 doctors which limited their ability to collect data. In attempts to address this, we limited the number of patients involved in each cycle to prevent a surplus of work. We also tried to collect data during weekend shifts when members of our team were on-call. Although this may have contributed to a slight bias of the results, it was the most pragmatic way to ensure collection of the data.

Despite addressing these issues, the proforma remains limited by the fact that it is user dependent and will only be effective if properly 


\section{BMJ Quality Improvement Reports}

completed. Furthermore, the data has only been from a narrow selection of medical wards and more widespread introduction would increase data reliability.

Future plans are for the proforma to be introduced into the Trust hospital stationary. In addition, by providing training on the proforma as part of the junior doctors induction programme in August 2014, they could be introduced as a routine occurrence in the hope of widespread acceptance.

\section{Conclusion}

We designed a Friday ward round proforma to compliment the current system of weekend handover, specifically focused on enhancing documentation of current plans and ceiling of care decisions for all medical patients. This had been highlighted as a concern by $\mathrm{F} 1$ doctors in the Trust. The proforma should prompt the junior doctors to specifically question about ceiling of care decisions from senior doctors, thereby preventing unnecessary treatment and investigations.

Our results demonstrated a significant improvement in both the time taken to understand a patients ceiling of care decision and the ease of doing so. There was an improvement from 153 seconds to 5 seconds for determining a ceiling of care decision (a 30-fold improvement), in patients who had completed proformas. The difficulty of understanding medical notes improved by $50 \%$ with the proformas.

The sheet has been reviewed for widespread distribution in the Trust by the documentation and nursing governance panels. We hope that use of these Friday ward round proformas will become commonplace throughout all medical specialities. The ward round proformas will feature in the induction week teaching for new F1 doctors to ensure their continued use.

\section{References}

1. Freemantle N, Richardson M, Wood J, Ray D, Khosla S, Shahian $\mathrm{D}$ et al. Weekend hospitalization and additional risk of death: an analysis of inpatient data. J R Soc Med 2012;105(2):74-84

2. British Medical Association. Safe handover: Safe patients. Guidance on clinical handovers for patients and managers. London: BMA, 2004.

3. Royal College of Physicians. Acute Care Toolkit 1: Handover. London: RCP, 2011.

4. Bhabra G, Mackeith S, Monterio P, Pothier D. An experimental comparison of handover methods. Ann R Coll Surg Engl 2007;89:298-300.

5. Desai SV, Feldman L, Brown L, Dezube R, Yeh HC, Punjabo N et al. Effect of the 2011 vs 2003 duty hour regulation-compliant models on sleep duration, trainee education, and continuity of patient care among internal medicine house staff: a randomized trial. JAMA Intern Med 2013;173(8):649-55.

6. General Medical Council. Tomorrow's doctors. Outcomes and standards for undergraduate medical education. London: GMC, 2009.

7. General Medical Council. Good Medical Practice. London: GMC, 2013.

8. The UK Foundation Programme Curriculum. Cardiff: UKFPO, 2012

9. Mehra A, Henein C. Improving hospital weekend handover: a user-centred, standardised approach. BMJ Qual Improv Report 2014;2:2.

10. Ashton C. Improving weekend patient handover. BMJ Qual Improv Report 2013;2:2.

11. Bethune R, Campbell K, Rose A, Wassall R, Price C, Siese $T$ et al. Improving weekend handover between junior doctors on medical and surgical wards. BMJ Qual Improv Report 2014;2:2.

12. Zarkali A, Black D, Smee E, Deshraj A, Smallwood N. Planning ahead: Improving escalation plans before the weekend. BMJ Qual Improv Report 2014;2:2.

13. Govier M, Metcalf P. Living for the weekend: electronic documentation improves patient handover. Clin Med 2012 Apr; 12(2): 124-7.

14. Till A, Sall H, Wilkinson J. Safe handover: Safe patients the electronic handover system. BMJ Qual Improv Report 2014;2:2.

15. Grewal B, Johnson F. To escalate or to palliate? An audit of patients at risk of deterioration on medical wards. BMJ Support Palliat Care 2014 Mar;4 Suppl 1:A81.

\section{Declaration of interests}

No competing interests. 